LBL-36640

UC-401

\title{
Energy States and Energy Flow Near the Transition States of Unimolecular Reactions
}

\author{
C. Bradley Moore \\ Department of Chemistry \\ University of California, Berkeley \\ and \\ Chemical Sciences Division \\ Lawrence Berkeley Laboratory \\ University of California \\ Berkeley, California 94720
}

October 1994

This work was supported by the Director, Office of Energy Research, Office of Basic Energy Sciences, Chemical Sciences Division, of the U.S. Department of Energy under Contract No. DE-AC03-76SF00098.

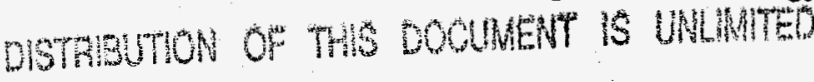




\section{DISCLAIMER}

This report was prepared as an account of work sponsored by an agency of the United States Government. Neither the United States Government nor any agency thereof, nor any of their employees, make any warranty, express or implied, or assumes any legal liability or responsibility for the accuracy, completeness, or usefulness of any information, apparatus, product, or process disclosed, or represents that its use would not infringe privately owned rights. Reference herein to any specific commercial product, process, or service by trade name, trademark, manufacturer, or otherwise does not necessarily constitute or imply its endorsement, recommendation, or favoring by the United States Government or any agency thereof. The views and opinions of authors expressed herein do not necessarily state or reflect those of the United States Government or any agency thereof. 


\section{DISCLAIMER}

Portions of this document may be illegible in electronic image products. Images are produced from the best available original document. 


\title{
CHAPTER
}

\section{ENERGY STATES AND ENERGY FLOW NEAR THE TRANSITION STATES OF UNIMOLECULAR REACTIONS*}

\author{
C. BRADLEY MOORE \\ Department of Chemistry, University of California, and \\ Chemical Sciences Division, Lawrence Berkeley Laboratory \\ Berkeley, California 94720
}

\section{Introduction}

Transition state theory (TST) as developed by Rice, Ramsperger, Kassel, and Marcus has been widely applied to unimolecular reactions. ${ }^{1-3}$ The RRKM theory is a microcanonical ensemble version of transition state theory. It incorporates the fundamental assumptions of TST that there is a local equilibrium between reactants and molecules crossing the transition state toward products along the reaction coordinate and that the molecule crossing through the transition state proceeds to products without recrossing. RRKM theory is based on the additional assumptions that all vibrational states in the excited molecule are equally probable and that vibrational energy flows freely among the different degrees of freedom at a rate much faster than the reaction rate. The RRKM rate constant for total energy $E$ and total angular momentum $\mathbf{J}$ is given by

$$
k(E, J)=\frac{W(E, J)}{h \rho(E, J)}
$$

where $W(E, J)$ is the number of energetically accessible states for vibration orthogonal to the reaction coordinate at the transition state, $\rho(E, J)$ is the density of vibrational states of the reactant, and $h$ is Planck's constant. RRKM theory presumes that motion along the reaction coordinate is classical and is decoupled from the bound vibrational motions at the transition state. The passage through the transition state is then vibrationally adiabatic, and the vibrational levels are defined over a sufficiently broad region near the transition state to give well-defined reaction thresholds or quantized channels connecting reactant to products. The rate constant is thus predicted to increase stepwise as the energy increases through each vibrational level or threshold at the transition state.

This step structure in $W(E, J)$ is apparently not an artifact of treating motion along the reaction coordinate classically since the quantum treatments of cumulative reaction probabilities $(\mathrm{CRP})^{4-6}$ exhibit quite similar structures. In his elegant treatment of the exact quantum theory of chemical reaction rates, Miller ${ }^{4}$ has expressed CRP as a sum of eigenvalues corresponding to the transition state theory sum of states, $N(E)$ in his work or $W(E, J)$ here. His paper shows that the stepped structure of the TST theory sum of states is found in the exact quantum CRP as a function of total energy for a single value of the total angular momentum. For bimolecular reactions this structure is smoothed out when summation over total angular momentum (integration over impact parameter in classical terms) is carried out. ${ }^{7}$ In practicable experiments further smoothing is

\footnotetext{
* Based on an address presented by C. Bradley Moore before "The Robert A. Weich Foundation, 38th Conference on Chemical Research, Chemical Dynamics of Transient Species," which was held in Houston, Texas, October 24-25, 1994.
} 
caused by averaging over the spread in relative translational energy of the reactants. However, for unimolecular reactions with reactant total energy (E) and total angular momentum (J) defined, the rate in Equation (1) is directly proportional to the exact quantum CRP or more approximately to $W(E, J)$ corrected for one-dimensional tunnelling through the barrier. Thus the influence of transition state structure and dynamics on reaction rates and product energy state distributions can be resolved most clearly for unimolecular reactions. With the resolution of modern laser experiments in cooled, pulsed jets it is not necessary to average over total angular momentum and energy. This suggests a form of spectroscopy in which the energies of the vibrational levels of the transition state are revealed by steps in the rate constant as a function of energy.

The transition state gives the initial conditions for the dynamics of energy release in the repulsive exit valley of the potential energy surface. 8,9 The peaks of product energy state distributions are determined by the geometry of the transition state and the shape of the potential energy surface in the exit valley. Distribution widths are determined by the distributions of atomic positions and momenta at the transition state, that is by the vibrational wavefunction of the molecule as it passes through the transition state. Consequently, if one looks in the wings of the distribution of a particular product quantum number one expects to see increases in product yield as the energy passes through the threshold for excitation of a transition state vibration which contributes strongly to that product degree of freedom. This can provide a dynamically biased spectroscopy useful for picking out energy levels for specific transition state vibrations. 10,11

The discussion above has implicitly assumed that the transition state, or point of no return, for a reaction is unambiguously located at the top of a single barrier along the reaction coordinate, a saddle point on the potential energy surface (Figure 1). There are important classes of unimolecular reactions which do not have this shape of PES. 12 For example, the rupture of a single bond to form two free radicals usually has no barrier to the recombination of those radicals and hence no saddle point or repulsive exit valley (Figure 2). 13 Thus there is a conceptual problem in defining and locating a transition state, if indeed one exists. The simplest choice is to place the transition state at the potential maximum along the reaction coordinate, at the products. This is the extreme limit of the loose transition state; $W(E, J)$ is given simply by the rotationvibration energy levels of the product molecules, phase space theory (PST).14 Exactly at threshold, only ground-state products, there is one and only one channel open and PST must give an accurate rate constant. However, as energy increases the number of product channels increases rapidly, in fact, much more rapidly than do the observed rate constants. This may be understood in terms of the increased spacing between energy levels as the product molecules approach and free rotations become hindered rotations, then torsions and ultimately bending vibrations of the reagent molecule. 3,15-17 If product channels are followed along the reaction coordinate back toward the stable reactant, one gets a family of non-crossing channel potentials for each symmetry. In the variational RRKM theory, 15,16 one locates the transition state by searching along the reaction coordinate for the point at which the number of open channels at a given energy is a minimum. This locates the transition state where the reactive flux is most limited; the transition state moves in along the reaction coordinate as the total energy increases above threshold. The statistical adiabatic channel model (SACM) ${ }^{17}$ uses these same potential curves and calculates the reaction rate constant by counting the number of channels with maxima at or below the total energy available. 


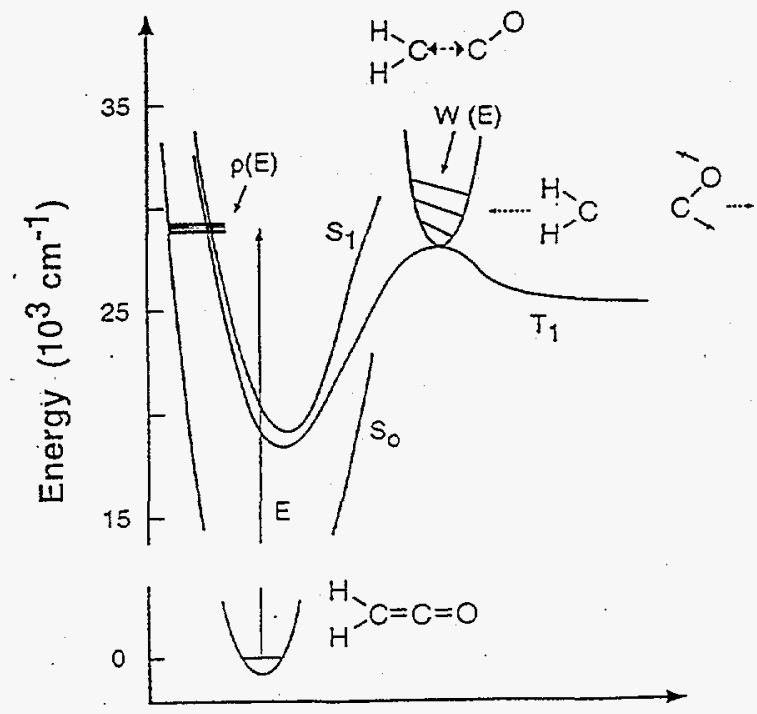

Reaction Coordinate

FIGURE 1

Dissociation of triplet ketene. The reaction coordinate is the distance between the carbon atoms. The solid lines represent the PES for the ground singlet $\left(S_{0}\right)$, the first excited singlet $\left(S_{1}\right)$, and the first triplet $\left(T_{1}\right)$ electronic states. The transition state, which separates the highly vibrationally excited reactant from the products, is depicted as a single potential well perpendicular to the reaction coordinate. This well is meant to represent the eight bound vibrational coordinates at the transition state.

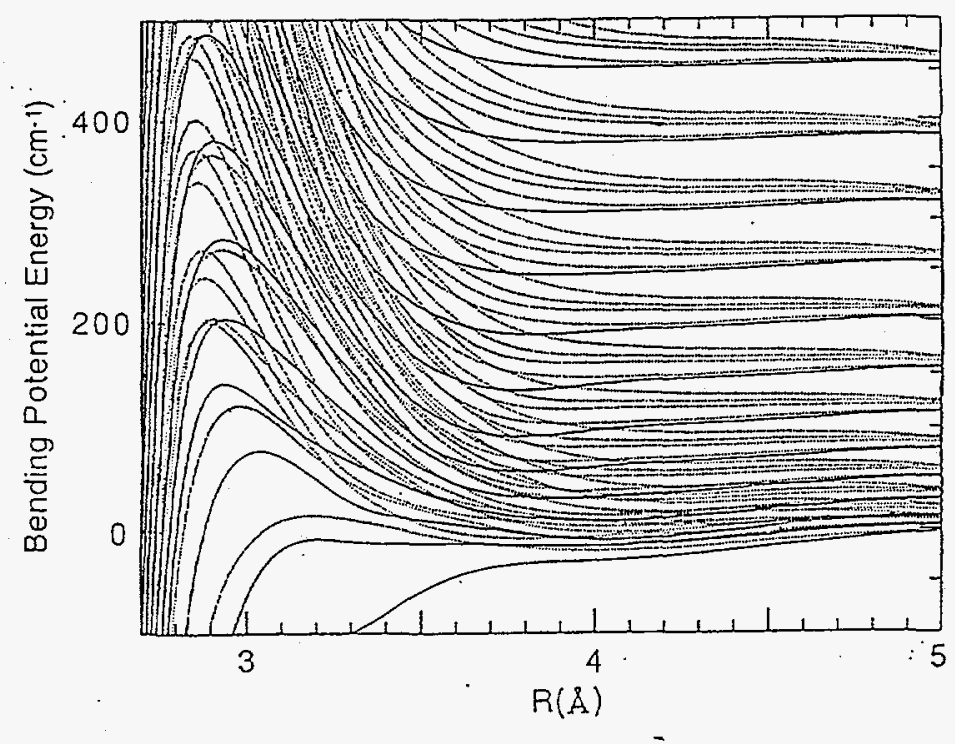

FIGURE 2

Adiabatic channels for a reaction with no barrier to recombination. Potential energy curves are shown for the energy levels of the bending vibrational motion of $\mathrm{NO}_{2}$ for the lowest potential surface. The dotted lines show those for the excited state potential surfaces dissociating to the $\mathrm{NO}\left({ }^{2} \Pi_{1 / 2}\right)+\mathrm{O}\left({ }^{3} P_{2}\right)$ limit. 13 
In practice these two theories give almost identical rates even though the postulated dynamics are somewhat different. Since there is no repulsive exit valley to push the product fragments apart rapidly, the dynamics of energy flow as the molecule moves along the reaction coordinate between the transition state and the separated product fragments can be complicated. For total energies sufficiently close to threshold that PST is valid, the product energy state distributions must be statistical, with all open channels equally probable. The rate constants must likewise increase stepwise at the threshold energy for each product energy state.

\section{Vibrational Level Thresholds at the Transition State for Bond Breaking over a Barrier}

The stepwise increase in rate constant with energy implied by Equation (1) has been observed for the dissociation of ketene over a barrier on its triplet PES to the triplet ground state of methylene and CO. 10,11

$$
\mathrm{CH}_{2} \mathrm{CO} \rightarrow{ }^{3} \mathrm{CH}_{2}+\mathrm{CO}
$$

This process ${ }^{11}$ is diagrammed in Figure 1 along with a cartoon of the ab initio geometry of Allen and Schaefer 18 of ketene at this transition state. The $a b$ initio calculations predict that the lowest frequency is for the torsion about the $\mathrm{C}-\mathrm{C}$ bond and that the $\mathrm{CCO}$ bend and $\mathrm{CH}_{2}$ out-of-plane wag are the next lowest. Hence, these modes are expected to dominate the structure of the rate constant $v s$. energy in the first few hundred wavenumbers above threshold. Ketene absorbs uv radiation in this energy region due to the $\mathrm{S}_{0} \rightarrow \mathrm{S}_{1}$ transition dipole moment. Electronic-vibration coupling, internal conversion and intersystem crossing, are sufficiently strong that the wavefunction of the excited molecule may be considered to be a statistical mixture of $S_{0}, T_{1}$ and $S_{1}$ basis states of comparable total energy. The density of vibrational states, the sum of that for all three electronic states, is essentially that of the ground state. The uv spectrum is smooth and flat. 19,20 Thus as a uv exciting laser is tuned, it produces a constant number of hot ketene molecules ready to react along any accessible channel.

The rate constant data of Figure 3 were obtained by uv excitation of ketene cooled to $4 \mathrm{~K}$ in a pulsed jet. 11 A vuv pulse probed the $C O$ product over a range of delay times to measure the product rise time. To confirm the observed structure in $\mathrm{k}(E)$, a photofragment excitation spectrum (PHOFEX) was measured using a short delay time so that the signal was proportional to the reaction rate constant as well as to the fraction of product formed in the $\mathrm{CO}(\mathrm{J})$ state probed. For the first $200 \mathrm{~cm}^{-1}$ above threshold, in which the $C O(\mathrm{~J})$ distribution does not change, the PHOFEX spectra show steps in the identical places as $\mathrm{k}(\mathrm{E})$ (Figure 4). The observed thresholds, Table 1, give barrier heights above product zero-point energies of $1281 \pm 15 \mathrm{~cm}^{-1}$ for $\mathrm{CH}_{2} \mathrm{CO}$ and $1071 \pm 40$ $\mathrm{cm}^{-1}$ for $\mathrm{CD}_{2} \mathrm{CO}$. The implied difference in zero-point energies at the transition state is $1096 \pm 20$ $\mathrm{cm}^{-1}$ compared to the theoretical prediction of $1091 \mathrm{~cm}^{-1}$. The barrier height is some $40 \%$ less than $a b$ initio calculation. 10,11

The torsion about the $\mathrm{C}-\mathrm{C}$ bond has a barrier height within the energy range of these experiments and thus must be treated carefully as a hindered rotor coupled to the free rotation about the $\mathrm{C}-\mathrm{C}$ bond. Figure 5 shows the potential curve and corresponding energy levels which give the best fit of RRKM theory to the observed $k(E)$, such as in Figure 3. The positions of many steps for $\mathrm{CH}_{2} \mathrm{CO}$ and $\mathrm{CD}_{2} \mathrm{CO}$ are fit with a two-parameter potential function. The torsional barrier of $240 \mathrm{~cm}^{-1}$ is $35 \%$ less than that calculated ab initio. 


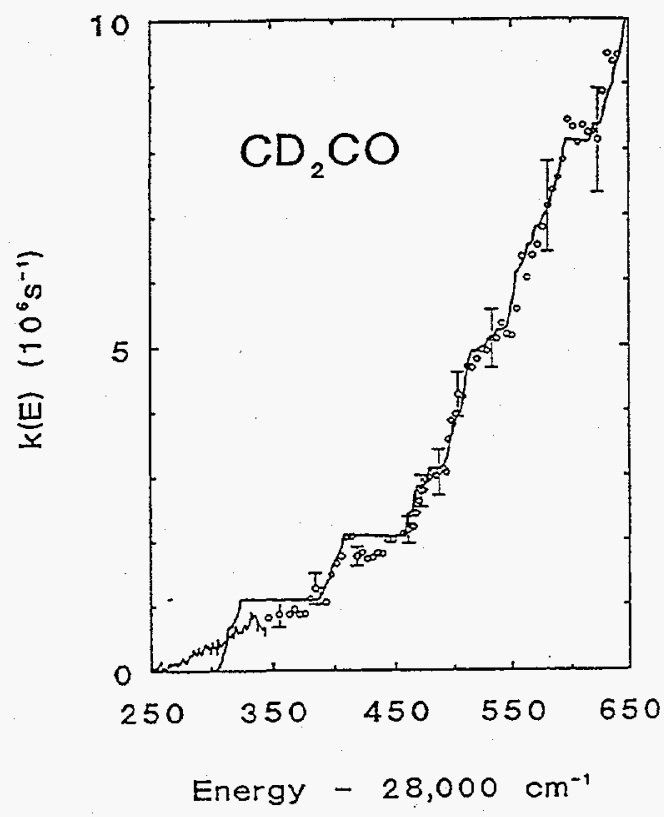

FIGURE 3

Stepped structure in the rate constant for $\mathrm{CD}_{2} \mathrm{CO}$ dissociation as a function of the photolysis energy. The $C O(v=0, J=12)$ PHOFEX curve $(\Delta t=1.7 \mu \mathrm{s})$ is shown in the reaction threshold region; its intensity is arbitrarily scaled. The solid line is a RRKM fit using the parameters in Table 1.11

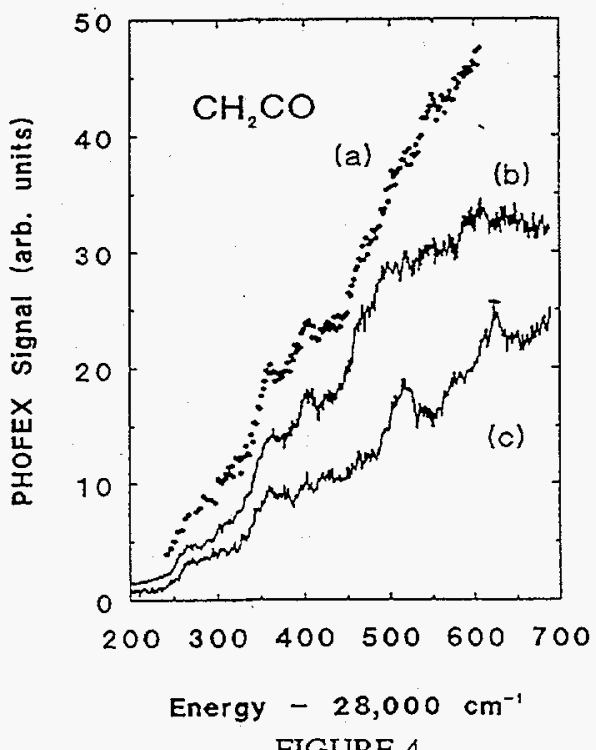

FIGURE 4

Dynamically biased spectroscopy of a transition state for dissociation. (a) PHOFEX curve calculated from the measured rates for $\mathrm{CH}_{2} \mathrm{CO} \rightarrow{ }^{3} \mathrm{CH}_{2}+\mathrm{CO}$ assuming that the distribution of $\mathrm{CO}(\mathrm{J})$ states is independent of energy. (b) PHOFEX spectrum at the peak of the CO(J) distribution, $\mathrm{CO}(\mathrm{J}=12)$. Evidently, the fraction of $\mathrm{CO}(J=12)$ begins to decrease at about 28,500 $\mathrm{cm}^{-1}$ where this curve flattens out. (c) PHOFEX spectrum for the wing of the $\mathrm{CO}(\mathrm{J})$ distribution, $\mathrm{CO}(J=2)$. Peaks show the energies of levels with one quantum of $\mathrm{CCO}$ bending excitation at the transition statc. The delay time of $50 \mathrm{~ns}$ for these curves is short compared to the time for dissociation to be complete. ${ }^{11}$ 
TABLE 1. Transition state information from the RRKM fits to experimental results. ${ }^{a}$

\begin{tabular}{ccc}
\hline & $\mathrm{CH}_{2} \mathrm{CO}$ & $\mathrm{CD}_{2} \mathrm{CO}$ \\
\hline$E_{\text {th }}$ & $28,250(10) \mathrm{cm}^{-1}$ & $28,310(15) \mathrm{cm}^{-1}$ \\
$v_{\mathrm{im}}{ }^{\mathrm{b}}$ & $100(40) i \mathrm{~cm}^{-1}$ & -- \\
$g_{f}{ }^{c}$ & $3.34(0.34)$ & $3.56(0.20)$ \\
$V_{0}$ & $240(30) \mathrm{cm}^{-1}$ & $240(30) \mathrm{cm}^{-1}$ \\
$V_{1}$ & $20(20) \mathrm{cm}^{-1}$ & $20(20) \mathrm{cm}^{-1}$ \\
$v_{7}^{*}(\mathrm{C}-\mathrm{C}-\mathrm{O}$ bend $)$ & $250(15) \mathrm{cm}^{-1}$ & $\mathrm{~d} 185(20) \mathrm{cm}^{-1}$ \\
$v_{8}^{*}\left(\mathrm{CH}(\mathrm{D})_{2}\right.$ wag $)$ & $290(15) \mathrm{cm}^{-1}$ & $\mathrm{~d} 240(20) \mathrm{cm}^{-1}$ \\
\hline \hline
\end{tabular}

a. The values in parentheses are uncertainties obtained from the fits.

b. The imaginary frequency $\left(v_{\mathrm{im}}\right)$ is used only for the fit to the first step in $\mathrm{k}(\mathrm{E})$ for $\mathrm{CH}_{2} \mathrm{CO}$ dissociation.

c. $\rho(E)=\rho_{W R}(E) \times f$. The $\rho_{W R}(E)$ is the density of vibrational states of reactant calculated from the Whitten-Rabinovitch approximation; $\rho_{W R}(E)=1.36 \times 10^{4}\left(/ \mathrm{cm}^{-1}\right)$ for $\mathrm{CH}_{2} \mathrm{CO}\left(\mathrm{S}_{0}\right)$ and $4.78 \times 10^{4}\left(/ \mathrm{cm}^{-1}\right)$ for $\mathrm{CD}_{2} \mathrm{CO}\left(\mathrm{S}_{0}\right)$ at $\mathrm{E}=28,500 \mathrm{~cm}^{-1}$.

d. Assignments are tentative.

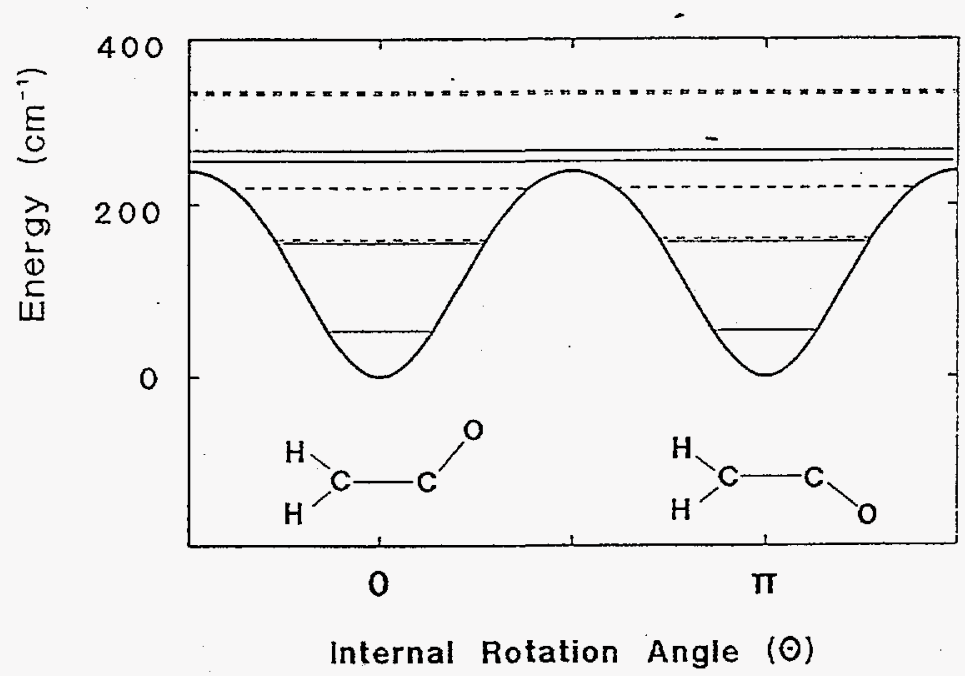

FIGURE 5 -

The potential function for hindered internal rotation of the transition state for triplet ketene, $V(\theta)=$ $(1 / 2) V_{0}(1-\cos 2 \theta)+(1 / 2) V_{1}(1-\cos 4 \theta)$, where $V_{0}=240 \mathrm{~cm}^{-1}$ and $V_{1}=20 \mathrm{~cm}^{-1}$. The $a b$ initio transition-state geometry is drawn. At the maximum, the $O$ is out of the plane with the CCO plane bisecting the $\mathrm{HCH}$ angle. The calculated energy levels are shown for $\mathrm{K}=0$ ( - , ortho; ---, para). The zero-point level splitting is less than $1 \mathrm{~cm}^{-1} .11$ 
The CCO bending vibration was identified by dynamically biased PHOFEX spectroscopy. Figure 4 shows PHOFEX spectra at the center of the $C O(J)$ distribution, $\mathrm{J}=12$, and in the wing at $\mathrm{J}=2$. Molecules coming through the transition state with one quantum of $\mathrm{CCO}$ bend excited have a $\mathrm{CO}(\mathrm{J})$ distribution with two maxima symmetrically displaced from $\mathrm{J}=12.11$ Such molecules are detected with strong preference at $\mathrm{J}=2$. The distinct peak observed at $250 \mathrm{~cm}^{-1}$ for $\mathrm{CH}_{2} \mathrm{CO}$ PHOFEX of $\mathrm{CO}(\mathrm{J}=2)$ matches the $a b$ initio frequency for the $\mathrm{CCO}$ bend of $252 \mathrm{~cm}^{-1}$ and is so assigned. The second peak occurs at the combination of the first excited torsional level with the $\mathrm{CCO}$ bend. The $\mathrm{CH}_{2}$ wag was identified with an otherwise unassigned step at $290 \mathrm{~cm}^{-1}$; this is significantly lower than the $a b$ initio value of $366 \mathrm{~cm}^{-1}$. These bending frequencies were identified at 185 and $240 \mathrm{~cm}^{-1}$ in $\mathrm{CD}_{2} \mathrm{CO}$ and may be significantly mixed and perturbed.

In the application of RRKM theory it is usually assumed not only that all vibrational degrees of freedom of a molecule are strongly mixed but also that Coriolis coupling mixes in the $\mathrm{K}_{\mathrm{a}}$ degree of freedom. ${ }^{3}$ To test this hypothesis for ketene, measurements were made as a function of jet temperature. For the cold ground state at $4 \mathrm{~K}$, several $\mathrm{J}$ levels are populated but only the lowest $K_{a}$ levels of each nuclear spin symmetry, $K_{a}=0,1$, are populated. When the molecule is excited, strong $\mathrm{K}_{\mathrm{a}}$ mixing causes all $\mathrm{K}_{\mathrm{a}}$ values up to the limit of $\pm J$ to be equally populated. The associated energy locked up in rotation as the molecule crosses the transition state is $A^{\ddagger} K_{a}{ }^{2}$, where $\mathrm{A} \ddagger$ is the a-axis rotational constant at the transition state. At a jet temperature of $30 \mathrm{~K}$ this requires zero to many tens of $\mathrm{cm}^{-1}$ of additional energy to cross through any particular vibrational threshold. The result of $\mathrm{K}$-mixing would be a significant decrease in rate constant with increasing jet temperature. The observed rates were almost identical at 4 and $30 \mathrm{~K}$, while the RRKM model predicts differences of up to $20 \%$ for $\mathrm{K}_{\mathrm{a}}$ mixed. Thus for values of $\mathrm{J} \leq 6, \mathrm{~K}_{\mathrm{a}}$ appears to be a good quantum number for highly excited ketene. 11

The step heights in Figure 3 give the density of states for ketene, or more precisely the density divided by the effective triplet degeneracy at the transition state, $0 \leq \mathrm{g}_{\mathrm{l}} \leq 3$. It is not currently known how many of the three symmetries of triplet spin sublevels are coupled strongly to the singlet levels by intersystem crossing. The data for $\mathrm{CH}_{2} \mathrm{CO}$ are accurately fit by $\rho=3.34 \rho_{\mathrm{WR}} / \mathrm{gt}$ and for $\mathrm{CD}_{2} \mathrm{CO}$ by $\rho=3.56 \rho_{\mathrm{WR}} / \mathrm{g}_{\mathrm{t}}$, Table 1.11 These and other parameters derived for the transition state are shown in Table 1 . There is ample precedent for the density of levels of molecules of comparable size to be a factor of three to ten larger than $\rho_{W R}$ calculated using the Whitten-Rabinovitch 21 approximation. It will be necessary to understand and to be able to predict these unexpectedly large densities of states in order for RRKM theory to be completely quantitative and predictive.

There are significant discrepancies in detail apparent between Equation (1) and the data of Figures 3 and 4 . The slope of the step at threshold is rather gradual compared to those at higher energy. The $k(E)$ curves actually show peaks, whereas $W(E, J)$ corrected for 1-dimensional tunnelling can only increase monotonically with energy. Clearly motion along the reaction coordinate and its coupling to other degrees of freedom needs to be treated quantum mechanically and a detailed knowledge of the potential function in the region of the transition state obtained. The reaction discussed in the following section provides a dramatic illustration of the importance of quantized motion along the reaction coordinate.

Other limitations to the application of the classic form of RRKM theory to unimolecular reactions can arise when IVR is not complete. For bonds which break at relatively low energies, partially good vibrational quantum numbers may exist and lead to rate constants which depend on 
quantum state as well as on total energy. Striking dependencies of reaction rate on rotational and vibrational quantum numbers have been observed for HFCO. 22 Even when vibrational mixing is complete and the wavefunctions of individual vibrational energy levels are in the ergodic or chaotic limit, rate constants must vary from state-to-state in the limit where initial quantum states are fully resolved. 23 For formaldehyde, average dissociation rate constants agree with RRKM theory, but rates fluctuate by well over an order of magnitude from one individual level to the next. $24-26$ The observed distributions of rates quantitatively matched the predicted quantum statistical fluctuations. This provides an example of an RRKM molecule in the quantum-state-resolved limit. The rate constant for statistical molecules with overlapping resonances has been treated theoretically. 27

\section{Quantized Motion Along the Reaction Coordinate in the Region of an}

\section{Isomerization Barrier}

The thresholds for three different unimolecular reactions of ketene lie within an energy range of $2000 \mathrm{~cm}^{-1}$ (Figure 6). 28 These reactions occur on potential energy surfaces with qualitatively different shapes and exhibit distinctly different dynamics. The carbon atom exchange reaction passes through the highly strained three membered ring geometry of oxirene in which the carbon atoms are equivalent. 29,30 At energies well above threshold, the measured reaction rates are fit accurately by RRKM theory with the transition state frequencies equal to the $a b$ initio frequencies of oxirene (Figure 7). ${ }^{31}$ The in-plane motion of $\mathrm{O}$ parallel to the $\mathrm{C}=\mathrm{C}$ bond is taken to be the reaction coordinate. Near the threshold, the rate constant exhibits a series of distinct peaks and valleys (Figure 8). 28 The coarse structure shows peaks separated by some $80 \mathrm{~cm}^{-1}$ with rates decreasing by as much as a factor of 2.3 for an energy increase of less than $20 \mathrm{~cm}^{-1}$. Even the finest structures apparent in Figure 8 are completely reproducible.

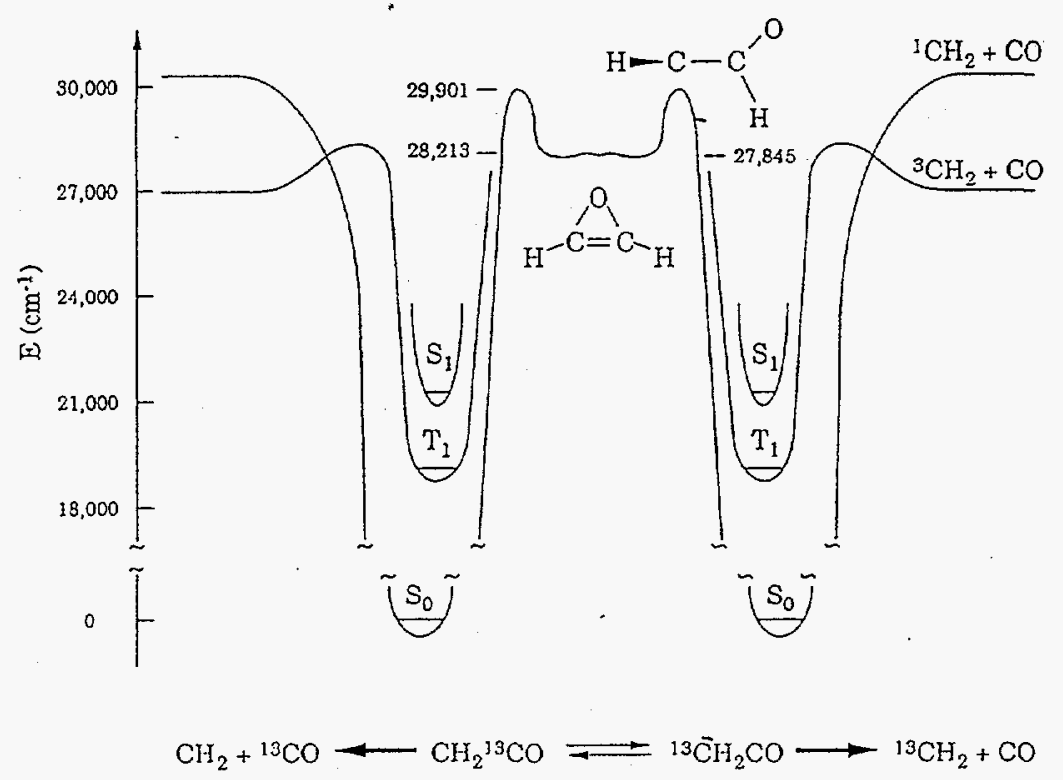

\section{FIGURE 6}

Potential energy diagram for all three unimolecular reactions of ketene. The structures and energies for the $\mathrm{C}$-atom exchange isomerization are from Reference 30. 


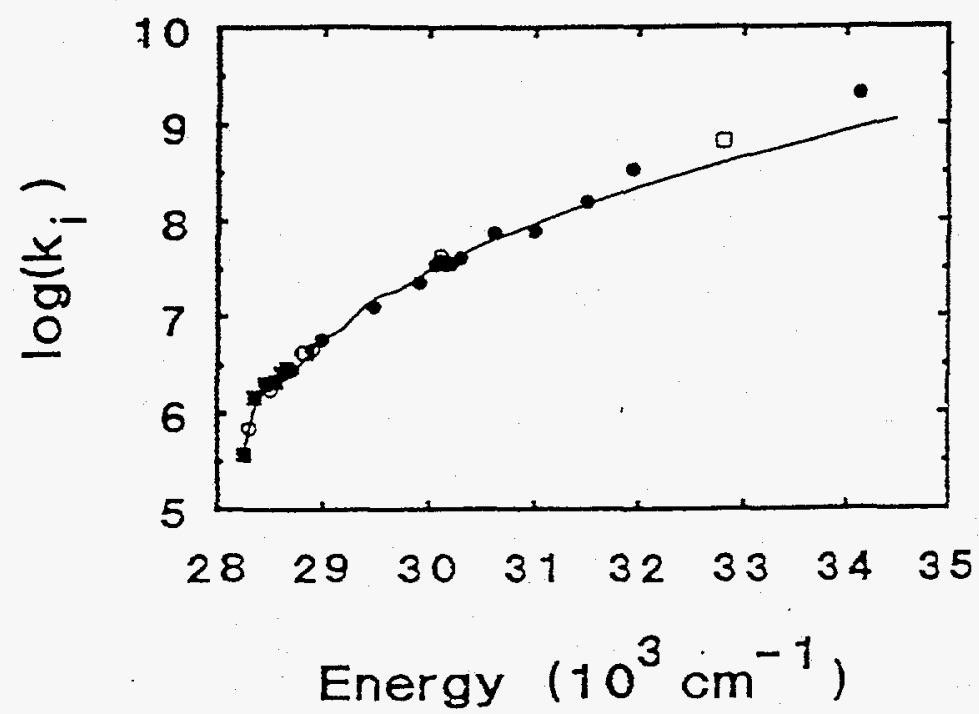

FIGURE 7

Rate constant for ketene isomerization, ${ }^{13} \mathrm{CH}_{2} \mathrm{CO} \rightarrow \mathrm{CH}_{2}{ }^{13} \mathrm{CO}$, as a function of excitation energy. The solid line is calculated with tunneling-corrected RRKM theory using an oxirene transition state, a threshold energy of $28360 \mathrm{~cm}^{-1}$, and a barrier imaginary frequency of 400 $i \mathrm{~cm}^{-1}$. Ab initio harmonic frequencies of oxirene from $\mathrm{G}$. Vacek, B. T. Colegrove, and H. F. Schaefer III, Chem. Phys. Lett., 177, 468 (1991) were used. 31

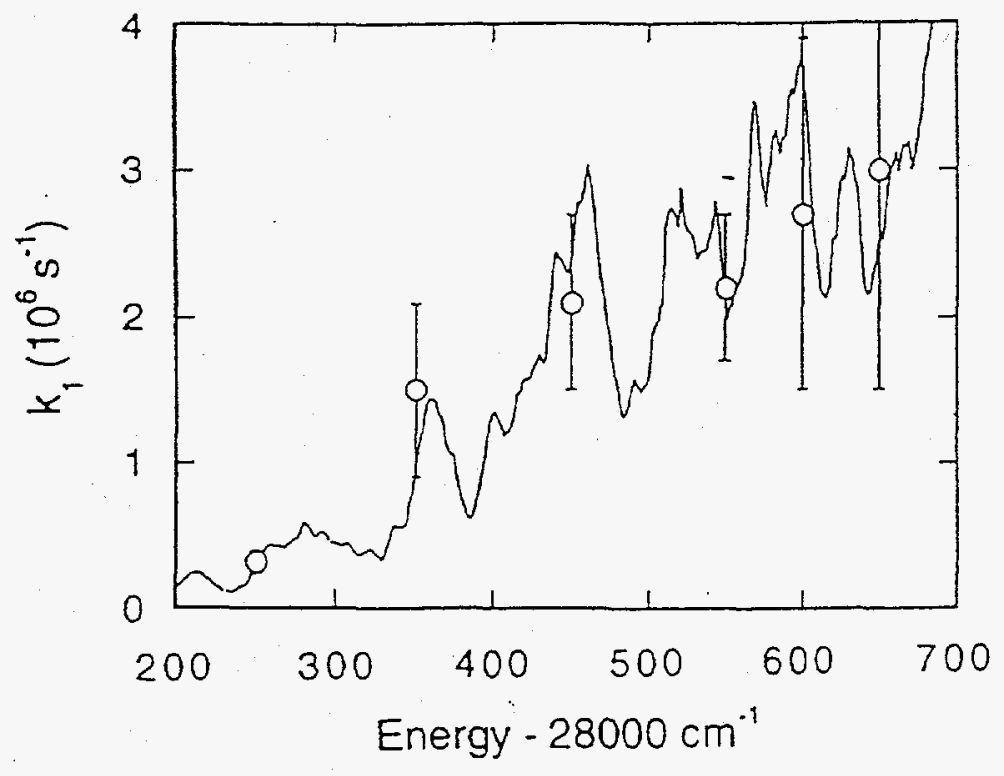

FIGURE 8

Rate constant for the isomerization ${ }^{13} \mathrm{CH}_{2} \mathrm{CO} \rightarrow \mathrm{CH}_{2}{ }^{13} \mathrm{CO}$ in the threshold region. The solid curve is from PHOFEX spectra of ${ }^{12} \mathrm{CO}$ and ${ }^{13} \mathrm{CO}$ products. All peaks of $0.2 \times 10^{-6} \mathrm{~s}^{-1}$ and larger are completely reproducible. The points are direct time evolution measurements with $2 \sigma$ error bars. 28 
This is the first example of such peaks in a rate constant. These peaks may be understood in terms of tunnelling through a barrier with a minimum at the top (Figure 6). 28 Quasi-stable resonances or vibrational levels exist inside the well for motion along the reaction coordinate. The tunnelling rate is greatly enhanced at the energies of these resonances. Resonances can also be expected above the top of the barrier since the-reaction coordinate changes from mostly O-atom motion in the central region of the barrier to mostly $\mathrm{H}$-atom motion at the transition states. At the energies of these resonances, motion along the reaction coordinate tends to be reflected at transitions between $\mathrm{H}$ motion and $\mathrm{O}$ motion. $A b$ initio calculations have established that there is a broad well at the top of the barrier which is about $2000 \mathrm{~cm}^{-1}$ deep. 29,30 It has been difficult to establish whether oxirene is a minimum or a saddle point on the surface at the center of this well. For dynamical calculations the important point is that oxirene is at the center of the barrier and very nearly at the bottom of the well within it. Quantum dynamical calculations in which the coordinates most closely coupled with the reaction coordinate are treated together in the region of the barrier reproduce the qualitative features of the observed rate data. 32 The structures in Figure 8 serve as a dramatic reminder that quantum mechanical resonances in the motion along the reaction coordinate and couplings between the reaction coordinate and other coordinates can be responsible for dramatic deviations from the classical motion along the reaction coordinate represented in Equation (1).

\section{The Variational Transition State: From Completely Loose to Tightly Constrained}

Singlet methylene is so reactive that it recombines with $\mathrm{CO}$ to form ketene without any barrier (Figure 6). Thus ${ }^{1} \mathrm{CH}_{2}$ is formed precisely at the threshold for ground state product formation, $30,116 \mathrm{~cm}^{-1} .19$ The reaction competes with dissociation to ${ }^{3} \mathrm{CH}_{2}$ and so the yield of product, PHOFEX signal in Figure 9, is directly proportional to the rate constant. 19,33,34 In the first few $\mathrm{cm}^{-1}$ there is only one state of singlet methylene energetically possible for each nuclear spin state (ortho and para states of the two $H$ nuclei are conserved throughout the dissociation process). 19 Thus the curve in Figure 9 is rigorously proportional to the rate constant for the first $20 \mathrm{~cm}^{-1}$. The PST calculation and the experiment are in precise agreement even to the summation over the thermally populated rotational states of ground-state ketene at $4 \mathrm{~K}$ in the jet. Thus the positions of the steps in $W(E, J)$ are exceptionally clear and within a fraction of $\mathrm{cm}^{-1}$ of the energy levels of the isolated product fragments. This is undoubtedly the sharpest spectrum showing the location of the energy levels of a transition state. More significantly, this provides an example of the limiting case of a completely loose transition state and the opportunity to study the transition from loose to tight as energy increases.

PST rates for the completely loose transition state are compared with the observed rates in Figure 10. The transition state begins to tighten in the range between 50 and $100 \mathrm{~cm}^{-1}$ above threshold. 20,33 The variational RRKM theory reproduces the experimental rate and has predicted product vibrational state populations accurately. 35,36 The product energy state distributions have been studied and found to be accurately given by PST up to $200 \mathrm{~cm}^{-1} .20$ The ${ }^{1} \mathrm{CH}_{2}$ becomes progressively colder compared to PST (though warmer absolutely) as energy increases. Up to an energy of $200 \mathrm{~cm}^{-1}$ the dynamics between the modestly tightened transition state and the separated products give fully randomized product energy state distributions. At higher energies the dynamics must become significantly adiabatic so that the product state distributions reflect to some extent the motions and energy levels at the transition state. However, this adiabaticity must be far from 


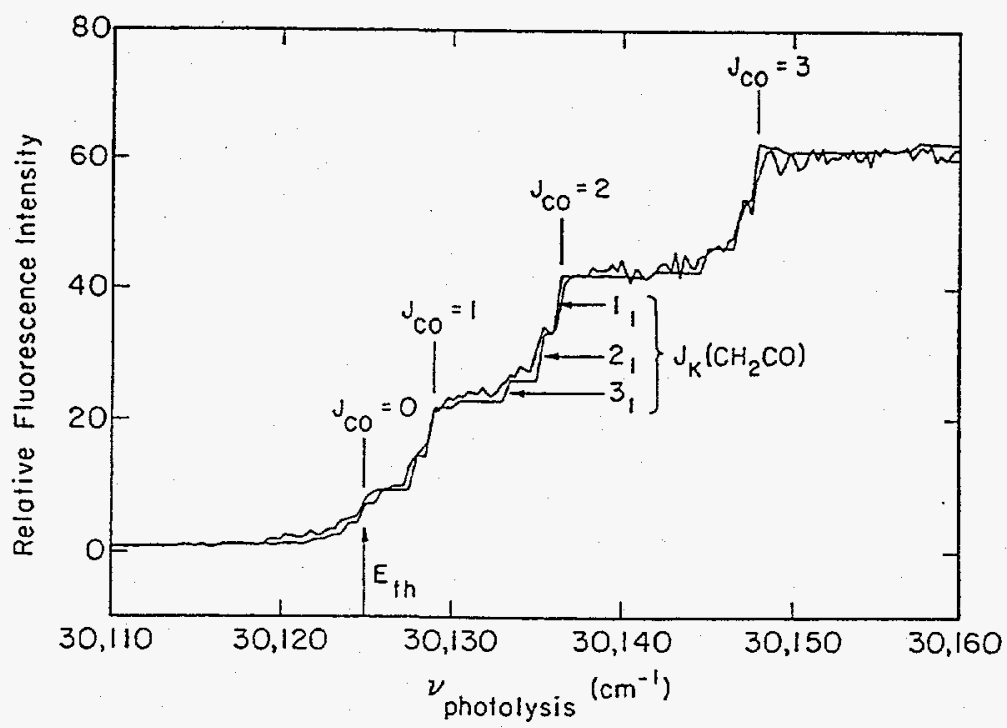

FIGURE 9

PHOFEX spectrum of the lowest rotational state of ortho singlet methylene near the threshold for $\mathrm{CH}_{2} \mathrm{CO} \rightarrow{ }^{1} \mathrm{CH}_{2}+\mathrm{CO}$. The smoother line is the phase-space theory rate constant. The step positions match the rotational energy levels for free $\mathrm{CO}$.

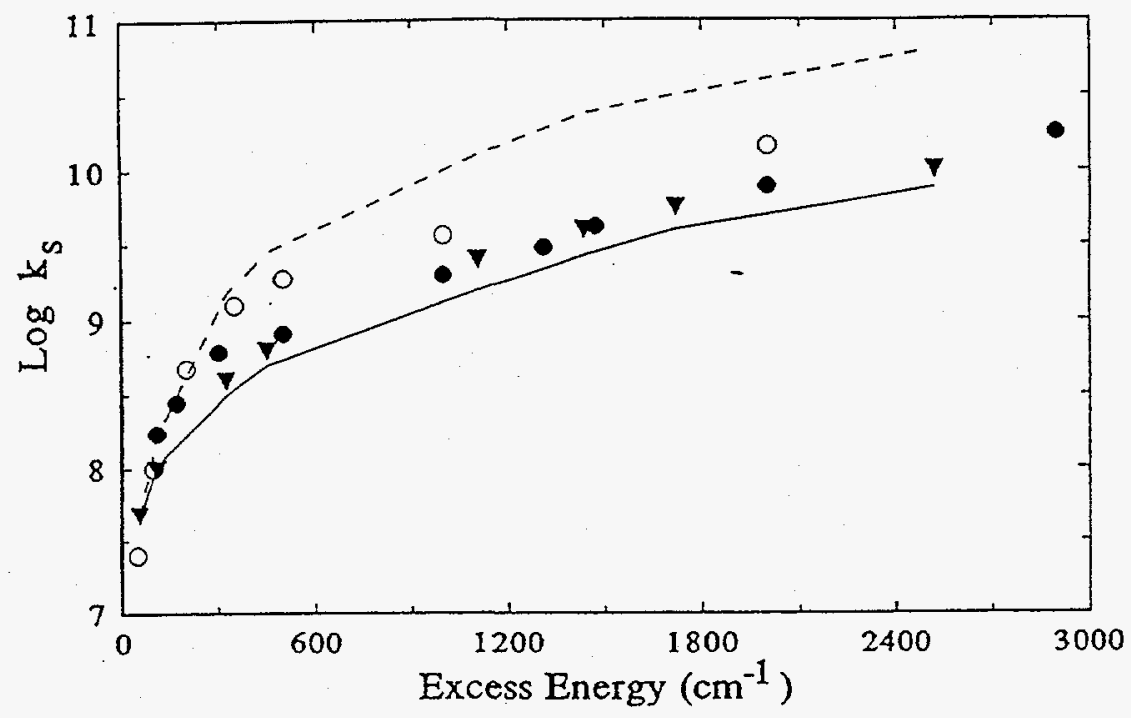

FIGURE 10

Energy dependence of the dissociation rate constant $\left(\mathrm{s}^{-1}\right)$ for $\mathrm{CH}_{2} \mathrm{CO} \rightarrow{ }^{1} \mathrm{CH}_{2}+\mathrm{CO}$ (first paper of Reference 20). The solid line is the singlet rate constant from the measured total rates [E. D. Potter, M. Gruebele, L. R. Khundkar, and A. H. Zewail, Chem. Phys. Lett., 164, 463 (1989)] and branching ratios (Reference 33 ). The filled triangles are variational-RRKM singlet rate constants (Reference 35). The open circles are a more recent variational RRKM calculation based on an ab initio PES (Reference 36). The filled circles are the PST rate constant for formation of the lowest ortho rotational state of ${ }^{l} \mathrm{CH}_{2}$ divided by the observed fractional population of that state. 
rigorous as each product energy state is observed precisely at its asymptotic energetic threshold. 19,20 Transitions from one adiabatic channel to another must occur quite frequently as the molecule evolves from the transition state to separated products. Such non-adiabatic dynamics may be induced by coupling with motion along the reaction coordinate, translation $\leftrightarrow$ rotationvibration energy transfer. It may also result from dynamics which hop between adiabats at narrowly avoided crossings. 37,38

The dissociation of $\mathrm{NO}_{2}$ to $\mathrm{NO}+\mathrm{O}$ is currently providing a wealth of information on the dynamics of reactions without a barrier. $13,39-42$ The structure in the PHOFEX spectrum of $\mathrm{NO}_{2}$ just above the reaction threshold is dominated by the features in the absorption spectrum. Miyawaki, Yamanouchi and Tsuchiya 39 have analyzed the widths of the overlapping resonances and obtained an approximate dissociation rate constant. They see that the opening of the channel for the first excited rotational state of $\mathrm{NO}$ approximately doubles the rate. The absolute rate at threshold, $0.85 \times 10^{10} \mathrm{~s}^{-1}$, agrees reasonably with PST when the density of states is taken from the fluorescence excitation spectrum just below threshold. 39,40 Katagiri and Kato 13 calculate that the energy maximum is at infinity for the first and second channels and moves in to $3.2 \AA$ and less

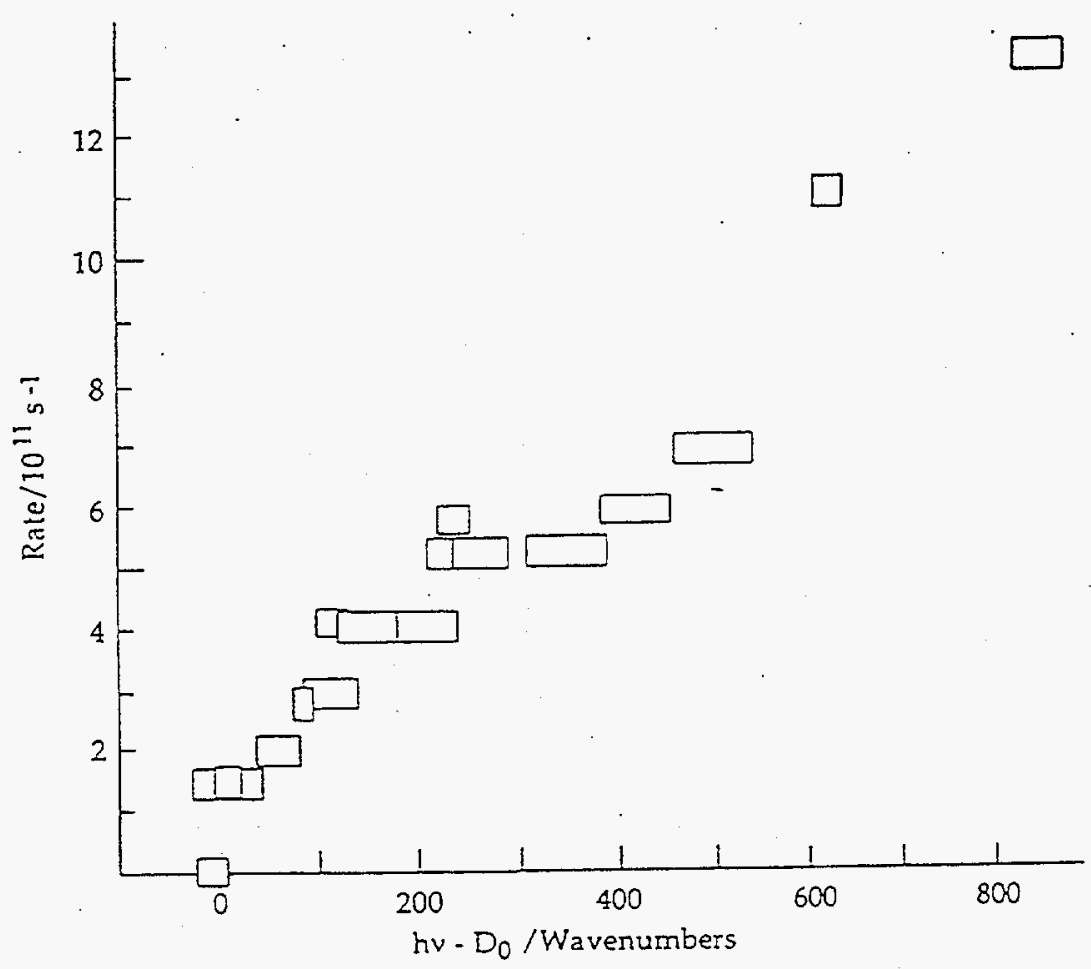

FIGURE 11

Unimolecular decay rate for $\mathrm{NO}_{2} \rightarrow \mathrm{NO}+\mathrm{O}$ vs. energy in excess of reaction threshold. The rectangle lengths represent the spectral bandwidths of the corresponding pump pulses. Statistical uncertainties lie within the rectangle heights. Step heights of about $1.6 \times 10^{11} \mathrm{~s}^{-1}$ are suggested (from the second paper of Reference 41). 
for the third and higher channels. That is to say that within the first ten $\mathrm{cm}^{-1}$ above threshold the transition state has tightened dramatically. Wittig and coworkers 41 measured rate constants with picosecond time resolution and 25 or $50 \mathrm{~cm}^{-1}$ of energy resolution (Figure 11). They reported much faster rate constants at threshold, $1.6 \times 10^{11} \mathrm{~s}^{-1}$, and steps with a spacing of about $100 \mathrm{~cm}^{-1}$ and a height comparable to the threshold rate. While the energy spacings are somewhat larger than those of the channel maxima predicted by Katagiri and Kato 13 in Figure 2, they are certainly suggestive of the spacings between bending levels of a variational transition state as it tightens. The increase in threshold rate constant between the linewidth and the picosecond data is a factor of about twenty. This suggests that the density of states for molecular motions inside the transition state decreases by something like an order of magnitude from the completely loose to the tight transition state. The tight transition state step height corresponds to the density of vibrational levels obtained spectroscopically near $16,000 \mathrm{~cm}^{-1}$ and extrapolated to the threshold near $25,000 \mathrm{~cm}^{-1}$ while that for the loose transition state is about an order of magnitude larger. 40 Highly anharmonic, large amplitude motions and coupling to the many electronically excited states which approach at the dissociation limit may cause this large density of states for the loose transition state. The energy dependence of product state distributions promises to provide detailed insight into the dynamics of energy flow during product separation and into the importance of the wavefunction of the initial molecular eigenstate or superposition of eigenstates in determining the final products. 42 These new experiments on $\mathrm{NO}_{2}$ introduce an element of caution into the denominator of Equation (1) and an element of confidence into the numerator in the application of the variational RRKM theory.

\section{Conclusion}

Understanding of unimolecular reactions has advanced rapidly in recent years. The use of lasers with jet-cooled samples has improved energy and angular momentum resolution for the reactant and time resolution for the rate constant by orders of magnitude. The resolution ofproduct quantum states has added a new dimension to unimōlecular dynamics. The concept of the transition state has sharpened greatly. In the past, the geometry, barrier height and vibrational frequencies of the transition state in RRKM theory were adjusted to fit thermal unimolecular reaction rate data. Usually these data could be described with three independent parameters and so chemists formed a comfortable view of the transition state as a method of rationalizing data and estimating the phase space available for reaction to various products. Now we understand that the concept of quantized vibrational level thresholds at the transition state is quantitatively meaningful. There have been successful quantitative tests of the ability of $a b$ initio theory to calculate transition state geometries accurately and barrier heights to a few $\mathrm{kJ} / \mathrm{mol}$ for simple molecules. Predicted frequencies tend to be somewhat too high for the softest modes which are of most importance in determining rates; however, the basic normal modes and sequence of frequencies seem to be correctly predicted. RRKM theory can be used with $a b$ initio results to predict rate constants to within a factor of two or three and may be used for quantitative extrapolation to conditions not accessible in the laboratory but important in practical situations. Experiments on single molecular eigenstates have revealed quantum statistical fluctuations in rates which are predicted quantitatively in the appropriate extension of RRKM theory. Many experiments seeking to demonstrate nonstatistical or non-RRKM dynamics have demonstrated the very wide range of applicability of the RRKM model. A few such experiments have demonstrated a lack of complete vibrational energy 
randomization in a reactant molecule. Dynamical theory has provided an exact quantum analog to RRKM theory which will combine with future experiments to define the extent to which quantized motion along the reaction coordinate and coupling between the reaction coordinate and vibrational degrees of freedom at the transition state are important. The future should also bring a much more complete understanding of the dynamics of energy flow as reaction products separate on a surface without a barrier to recombination.

\section{Acknowledgments}

I am pleased to acknowledge many stimulating discussions with and advance copies of results from Professors S. Klippenstein, W.H. Miller, H.F. Schaefer, III and S.C. Smith. The work in my own laboratory at Berkeley has been supported by the National Science Foundation under grants CHE8816552 and CHE9316640 and by the Chemical Sciences Division of the U.S. Department of Energy under contract DE-AC03-76SF0098. 


\section{REFERENCES}

1. R. A. Marcus and O. K. Rice, J. Phys. Colloid Chem., 55, 894 (1951); R. A. Marcus, J. Chem. Phys., 20, 359 (1952).

2. W. Forst, Theory of Unimolecular Reactions, Academic, New York (1973).

3. R. G. Gilbert and S. C. Smith, Theory of Unimolecular and Recombination Reactions, Blackwell, Oxford (1990).

4. W. H. Miller, Proceedings of The Robert A. Welch Foundation 38th Conference on Chemical Research, (to be published, 1994).

5. D. C. Chatfield, R. S. Friedman, D. G. Truhlar, B. C. Garrett, and D. W. Schwenke, J. Am. Chem. Soc., 113, 486 (1991).

6. T. Seideman and W. H. Miller, J. Chem. Phys., 97, 2499 (1992).

7. S. L. Mielke, G. C. Lynch, D. G. Truhlar and D. W. Schwenke, J. Phys. Chem., 98, 8000 (1994).

8. I-C. Chen and C. B. Moore, J. Phys. Chem., 94, 269 (1990).

9. R. Schinke, Photodissociation Dynamics; Cambridge Monographs on Atomic, Molecular and Chemical Physics, Vol 1, Cambridge University, New York (1993).

10. E. R. Lovejoy, S. K. Kim, and C. B. Moore, Science, 256, 1542 (1992).

11. S. K. Kim, E. R. Lovejoy, and C. B. Moore, J. Chem Phys., (submitted).

12. W. H. Green, C. B. Moore, and W. F. Polik, Annu. Rev. Phys. Chem., 43, 591 (1992).

13. H. Katagiri and S. Kato, J. Chem. Phys., 99, 8805 (1993).

14. P. Pechukas and J. C. Light, J. Chem. Phys., 42, 3281 (1965).

15. D. M. Wardlaw and R. A. Marcus, Adv. Chem. Phys., 70, 231 (1988).

16. D. G. Truhlar and B. C. Garrett, Annu. Rev. Phys. Chem., 35, 159 (1984).

17. M. Quack and J. Troe, Int. Rev. Phys. Chem., 1, 97 (1981).

18. W. D. Allen and H. F. Schaefer III, J. Chem. Phys., 89, 329 (1988); 84, 2212 (1986).

19. W. H. Green, I-C. Chen, and C. B. Moore, Ber. Bunsenges. Phys. Chem., 92, 389 (1988); I-C. Chen, W. H. Green, and C. B. Moore, J. Chem. Phys., 89, 314 (1988).

20. I. Garcia-Moreno, E. R. Lovejoy, and C. B. Moore, J. Chem. Phys., 100, 8890 and 8902 (1994).

21. G. Z. Whitten and B. S. Rabinovitch, J. Chem. Phys., 38, 2466 (1963).

22. Y. S. Choi and C. B. Moore, J. Chem. Phys., 97, 1010 (1992). 
23. W. F. Polik, C. B. Moore, and W. H. Miller, J. Chem. Phys., 89, 3584 (1988).

24. W. F. Polik, D. R. Guyer, and C. B. Moore, J. Chem. Phys., 92, 3453 (1990).

25. W. F. Polik, D. R. Guyer, C. B. Moore, and W. H. Miller, J. Chem. Phys., 92, 3471 (1990)

26. R. Hernandez, W. H. Miller, C. B. Moore, and W. F. Polik, J. Chem. Phys., 99, 950 (1993).

27. U. Peskin, H. Reisler, and W. H. Miller, J. Chem. Phys., (submitted);

U. Peskin, W. H. Miller and H. Reisler, J. Chem. Phys., (submitted).

28. E. R. Lovejoy and C. B. Moore, J. Chem. Phys., 98, 7846 (1993).

29. G. Vacek, J. M. Galbraith, Y. Yamaguchi, H. F. Schaefer, III, R. H. Nobes, A. P. Scott, and L. Radom, J. Phys. Chem., 98, 8660 (1994).

30. A. P. Scott, R. H. Nobes, H. F. Schaefer, III, and L. Radom, J. Am. Chem. Soc., (submitted).

31. E. R. Lovejoy, S. K. Kim, R. A. Alvarez and C. B. Moore, J. Chem. Phys., 95, 4081 (1991).

32. D. Gezelter and W. H. Miller, private communication.

33. S. K. Kim, Y. S. Choi, C. D. Pibel, Q.-K. Zheng, and C. B. Moore, J. Chem. Phys., 94, 1954 (1991).

34. W. H. Green, A. J. Mahoney, Q.-K. Zheng, and C. B. Moore, J. Chem. Phys., 94, 1961 (1991).

35. S. J. Klippenstein and R. A. Marcus, J. Chem. Phys., 91, 2280 (1989).

36. J. Yu and S. J. Klippenstein, J. Phys. Chem., 95, 9882 (1991).

37. E. I. Dashevskaya, E. E. Nikitin, and J. Troe, J. Chem. Phys., 973318 , (1992).

38. A. I. Maergoiz, E. E. Nikitin, and J. Troe, J.Chem. Phys., 95, 5117 (1991).

39. J. Miyawaki, K. Yamanouchi, and S. Tsuchiya, J. Chem. Phys., 99 254, (1993).

40. S. I. Ionov, H. F. Davis, K. Mikhaylichenko, L. Valachovic, R. A. Beaudet and C. Wittig, J. Chem. Phys., 101, 4809 (1994).

41. G. A. Brucker, S. I. Ionov, Y. Chen, and C. Wittig, Chem. Phys. Lett. 194, 301 (1992); S. I. Ionov, G. A. Brucker, C. Jaques, Y. Chen, and C. Wittig, J. Chem. Phys., 99, 3420 (1993).

42. S. A. Reid, J. T. Brandon, M. Hunter, and H. Reisler, J. Chem. Phys., 99, 4860 (1993); S. A. Reid, D. C. Robie, and H. Reisler, J. Chem. Phys., 100, 4256 (1994); S. A. Reid and H. Reisler, J. Chem. Phys., 101, 5683 (1994). 